\title{
Interactions of Single-Nozzle Sonic Propulsive Deceleration Jets on Mars Entry Aeroshells
}

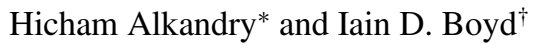 \\ University of Michigan, Ann Arbor, Michigan 48109 \\ and \\ Erin M. Reed,, \pm Joshua R. Codoni, \\ University of Virginia, Charlottesville, Virginia 22903
}

DOI: $\underline{10.2514 / 1.52232}$

\begin{abstract}
As the mass and landing site altitude of future Mars entry systems increase, the size requirements for conventional aerodynamic decelerators are becoming unfeasible. One option is propulsive decelerator jets. The use of propulsive decelerator jets, however, involves complex flow interactions that are still not well understood. This paper describes numerical and experimental techniques currently used to investigate these interactions. The paper also presents computational results for single-nozzle sonic propulsive decelerator jets. The numerical simulations use a scaled Mars Science Laboratory aeroshell in Mach 12 laminar flow of $I_{2}$-seeded $N_{2}$ gas. The results show that flowfield features, such as the bow and propulsive decelerator jet shocks, are affected by the thrust coefficient of the propulsive decelerator nozzle. These effects also extend to the surface and aerodynamic properties of the aeroshell. As the thrust coefficient increases, the pressure and shear stress approach roughly constant values over most of the aeroshell surface, and the drag coefficient decreases and approaches a constant value equal to approximately $8 \%$ of the value for the propulsive decelerator jet-off case. Finally, comparisons between the numerical results and experimental data show good agreement in the bow shock profile and standoff distance, as well as the aerodynamic properties of the aeroshell.
\end{abstract}

\section{Nomenclature}

$C_{D}=$ drag coefficient

$C_{f} \quad=$ coefficient of skin friction

$C_{P}=$ pressure coefficient

$C_{T}=$ thrust coefficient

$F_{D}=$ drag force, $\mathrm{N}$

$F_{T}=$ thrust force, $\mathrm{N}$

$K n=$ Knudsen number

$M=$ Mach number

$P \quad=\quad$ static pressure, $\mathrm{Pa}$

$q=$ dynamic pressure, $\mathrm{Pa}$

$R e=$ Reynolds number

$S \quad=$ aeroshell frontal area, $\mathrm{m}^{2}$

$U=$ velocity, $\mathrm{m} / \mathrm{s}$

$X=$ mole fraction

$\gamma=$ ratio of specific heats

$\rho=$ density, $\mathrm{kg} / \mathrm{m}^{3}$

$\tau=$ shear stress, $\mathrm{Pa}$

\section{Subscripts}

$\mathrm{amb}=$ post-bow-shock (ambient) conditions

jet $=$ propulsive decelerator jet conditions

Presented as Paper 2010-4888 at the 10th AIAA/ASME Joint Thermophysics and Heat Transfer Conference, Chicago Illinois, 28 June-1 July 2010; received 31 August 2010; revision received 11 March 2011; accepted for publication 11 March 2011. Copyright $\odot 2011$ by Hicham Alkandry, Iain D. Boyd, Erin M. Reed, Joshua R. Codoni, and James C. McDaniel. Published by the American Institute of Aeronautics and Astronautics, Inc., with permission. Copies of this paper may be made for personal or internal use, on condition that the copier pay the $\$ 10.00$ per-copy fee to the Copyright Clearance Center, Inc., 222 Rosewood Drive, Danvers, MA 01923; include the code 0022-4650/11 and $\$ 10.00$ in correspondence with the CCC.

*Graduate Student, Department of Aerospace Engineering. Student Member AIAA.

${ }^{\dagger}$ Professor, Department of Aerospace Engineering. Fellow AIAA.

${ }^{\star}$ Graduate Student, Department of Mechanical and Aerospace Engineering. Student Member AIAA.

${ }^{\S}$ Professor, Department of Mechanical and Aerospace Engineering. Associate Fellow AIAA ref $=$ reference freestream conditions

$0=$ total (stagnation) conditions

\section{Introduction}

$\mathbf{C}$ ONVENTIONAL aerodynamic decelerators for future Mars landers may be insufficient due to extremely large parachute size requirements. The Mars Science Laboratory (MSL) spacecraft, scheduled for launch in the Fall of 2011, has an estimated landing mass larger than $1700 \mathrm{~kg}$, which is far greater than the entry mass for any previous Mars entry system (e.g., Viking) [1]. The MSL will also land at a site that is up to $1 \mathrm{~km}$ above the reference altitude. Future missions, including possible human missions, may continue this trend of carrying more payload masses to Mars in order to conduct more sophisticated in situ experiments and landing at sites of scientific interest that are at higher altitudes. However, it is not possible to simply extend the Viking-heritage technology (e.g., supersonic diskgap-band parachutes and $70^{\circ}$ blunt-body aeroshells) to the dimensions and deployment conditions required by these missions [2]. These challenges may be resolved by using an additional propulsive decelerator (PD) component in order to slow the vehicle down to appropriate speeds.

Previous work on PD jets was conducted in the 1960s and early 1970s primarily through wind-tunnel experiments. This work mainly examined the aerodynamic effects of the PD jets for both singlenozzle and multinozzle configurations, which are shown in Figs. 1a and $1 \mathrm{~b}$, respectively. Experimental results showed that, for relatively low nondimensional nozzle thrust values, only a small augmentation of the axial force (the sum of the aerodynamic drag and the thrust forces) beyond that provided by the PD jet-off case was observed for the single-nozzle configuration [3]. Beyond the 1970s, however, there has been very little work on propulsive deceleration, and several important limitations still exist. These limitations include a lack of extensive experimental data and validated numerical approaches that can accurately and efficiently simulate the complex flow interactions that are generated in the use of PD jets.

This paper will describe numerical and experimental approaches that are used to understand the complex flow interactions between the PD jets, the freestream, and the aeroshell. It will also present numerical results using a scaled MSL aeroshell with a sonic PD jet in 


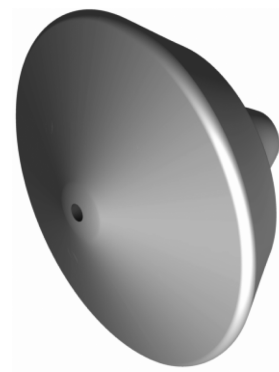

a) Single nozzle

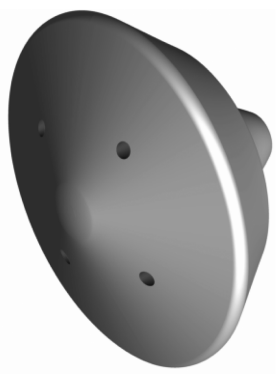

b) Multinozzle
Fig. 1 PD jet configurations.

a single-nozzle configuration located at the center of the forebody. These results are presented in four parts. The first, second, and third sections of this paper will focus on the effects of the PD jet on the flowfield, surface, and aerodynamic properties of the aeroshell, respectively. In the last section of the paper, comparisons between numerical and experimental results will be presented to assess the computational method.

\section{Technical Approach}

\section{A. Experimental Technique}

Experimental measurements are obtained using the planar laserinduced iodine fluorescence (PLIIF) technique at a hypersonic windtunnel facility at the University of Virginia. The PLIIF technique is a nonintrusive, spatially resolved, time-averaged optical method for measurements in hypersonic, rarefied flows. The technique has been used for both qualitative and quantitative measurements [4-6]. PLIIF involves seeding iodine into a flowfield and exciting the iodine molecules to a higher energy with an argon ion laser. The laser beam is turned into a thin laser sheet and passed through the flowfield of interest. The resulting fluorescence is imaged at $90^{\circ}$ using a cooled scientific-grade charge-coupled device (CCD) camera. Measurements of the absorption spectrum are made as the laser is tuned in frequency. By fitting the measured absorption spectra at every point in the flowfield, the velocity, temperature, and injectant mole fraction can be deduced. The technique provides qualitative flow visualization images when the laser is operated in the broadband mode (laser gain profile much wider than iodine absorption linewidth). The results to be presented herein have been taken with this approach.

The hypersonic flow facility at the University of Virginia is capable of providing Mach numbers and Knudsen numbers up to 16 and 1, respectively. Hypersonic flow from an underexpanded jet is produced by the expansion of iodine-seeded nitrogen gas across a thin circular orifice of diameter $D=2 \mathrm{~mm}$ into a continuously evacuated vacuum chamber. The stagnation pressure and temperature in the wind tunnel are $1.8 \mathrm{~atm}$ and $300 \mathrm{~K}$, respectively. Figure $2 \mathrm{a}$ presents a schematic of the experimental setup in the hypersonic flow facility. Figure 2b shows calculated Mach number and Knudsen number $K n$ variations inside the freejet facility [4]. These contours show the barrel shock that develops at the entrance of the test section and terminates at the Mach disk. Models are placed in the underexpanded jet core for testing at hypersonic conditions. The freestream Mach number and flow properties can be changed by adjusting the distance of the test model to the orifice.

\section{B. Numerical Method}

Numerical simulations are performed using the computational fluid dynamics (CFD) code LeMANS, developed at the University of Michigan for simulating hypersonic reacting flows [7-9]. This general-purpose three-dimensional parallel code solves the laminar Navier-Stokes equations on unstructured computational grids, including thermochemical nonequilibrium effects with second-order accuracy. The flow is modeled assuming that the continuum approximation is valid. Furthermore, it is assumed that the translational and rotational energy modes of all species can be described by two different temperatures, $T_{\text {tra }}$ and $T_{\text {rot }}[10]$, respectively, while the vibrational energy mode and electron energy of all species can be described by a single temperature $T_{\text {vib }}$. The electronic energy is neglected due to the relatively small temperatures achieved in the hypersonic flows of interest. In LeMANS, the mixture transport properties can be computed using several options. In this study, Wilke's semiempirical mixing [11] is used with species viscosities calculated using Blottner et al.'s model [12] and species thermal conductivities determined using Eucken's relation [13].

The finite volume method applied to unstructured grids is used to solve the set of partial differential equations. LeMANS can simulate two-dimensional or axisymmetric flows using any mixture of quadrilateral and triangular mesh cells, and it can simulate threedimensional flows using any mixture of hexahedra, tetrahedra, prisms, and pyramids. A modified Steger-Warming flux vector splitting scheme is used to discretize the inviscid fluxes across cell faces, which is less dissipative and produces better results in boundary layers compared with the original scheme [14]. The viscous terms are computed using cell-centered and nodal values. Time integration is performed using either a point implicit or a line implicit method. LeMANS is parallelized using METIS [15], which is a software package for partitioning large computational meshes, and message passing interface to communicate the necessary information between processors.
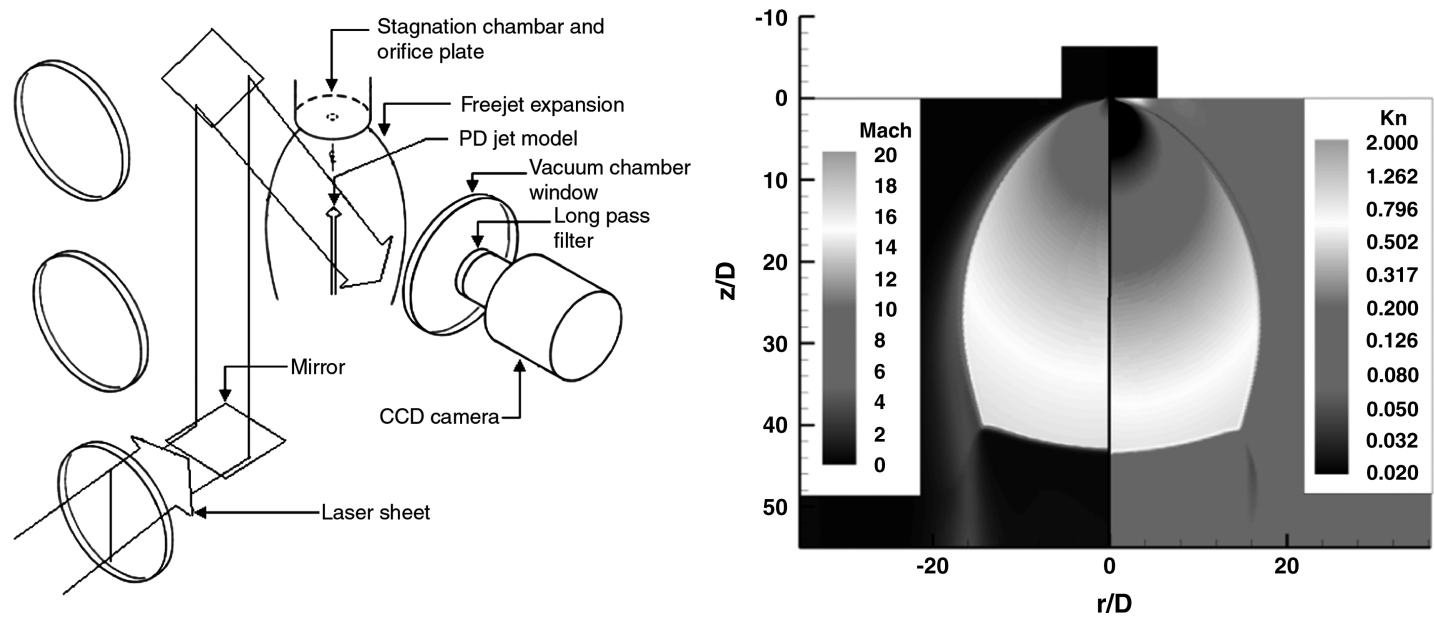

a) Schematic of the experimental setup

b) Calculated mach number and knudsen number contours in the test section [4]

Fig. 2 Experimental facility. 

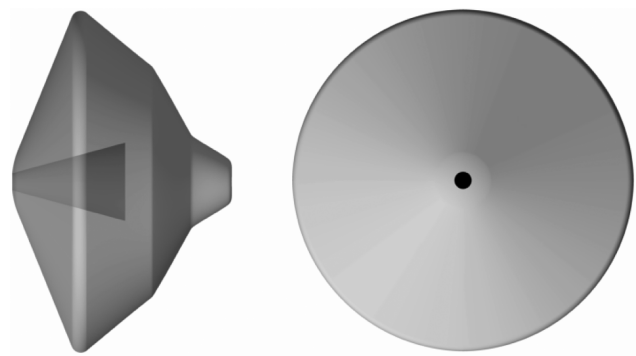

a) Side view

b) Front view
Fig. 3 Model geometry.

\section{Numerical Setup}

The geometry of the model used in this study is shown in Fig. 3 . The model is $10 \mathrm{~mm}$ in diameter, which is equivalent to approximately $0.22 \%$ of the size of the MSL aeroshell. The PD nozzle is located at the center of the forebody and consists of a converging section, with a nozzle exit diameter of $0.5 \mathrm{~mm}$. A sonic nozzle is chosen, because it provides a good reference frame from which to begin other studies and it is the easiest to machine for the experimental models. The discharge coefficient of the nozzle, defined as the ratio of actual to ideal mass flow rate, is equal to 0.92 .

To accurately simulate the flow in the experimental facility, $\mathrm{I}_{2}$-seeded $\mathrm{N}_{2}$ gas is used in the numerical simulations with a seeding ratio of $200 \mathrm{ppm}$. The vibrational temperature is assumed frozen at the stagnation value of $300 \mathrm{~K}$. The freestream rotational temperature is assumed to be equal to the translational temperature. Radially nonuniform conditions based on the freejet relations of Ashkenas and Sherman [16] are also used as flow conditions input to LeMANS at the upstream boundary. A previous study showed that these nonuniform freestream conditions widen the bow shock around the aeroshell and decrease the drag coefficient by $6.4 \%$ compared with uniform conditions [17]. The Mach number at a distance $z$ away from the orifice along the centerline of the freejet is given by Eq. (1):

$$
M=A\left(\frac{z-z_{0}}{D}\right)^{\gamma-1}-\frac{1}{2}\left(\frac{\gamma+1}{\gamma-1}\right)\left[A\left(\frac{z-z_{0}}{D}\right)^{\gamma-1}\right]^{-1}
$$

where $D$ is the diameter of the freejet orifice, and $A$ and $z_{0} / D$ are constants determined for values of $\gamma$ and are equal to 3.65 and 0.40 , respectively, for $\gamma=1.4$. All other fluid properties along the freejet axis can be computed using the Mach number defined in Eq. (1), the stagnation conditions in the wind tunnel, and the isentropic relations. The density distribution at a fixed distance from the orifice exit is a function of the streamline angle $\theta$ with respect to the freejet axis, as shown in Eq. (2):

$$
\frac{\rho(\theta)}{\rho(0)}=\cos ^{2}\left(\frac{\pi \theta}{2 \Phi}\right)
$$

where $\Phi$ is also a constant determined for each value of $\gamma$ and is equal to 1.662 for $\gamma=1.4$. For this study, a reference freestream Mach number of 12 is used in order to minimize the interaction of the bow shock around the aeroshell and the barrel shock created in the test section in the experiments by pushing the triple point (point of interaction) downstream of the model. As a result, it is not necessary to model the entire test section of the wind tunnel in the numerical simulations, which dramatically cuts down on the computational cost and complexity. Figure 4, modified from McDaniel et al. [4], shows a to-scale plot of the location of the aeroshell model with respect to the freejet orifice and velocity streamlines for the Ashkenas and Sherman boundary conditions [16]. A set of reference freestream conditions is obtained using isentropic relations for a reference freestream Mach number of 12 . These reference conditions are presented in Table 1 and are used to compute nondimensional quantities, such as the drag coefficient.

The boundary conditions for the PD jet are computed such that sonic conditions are obtained at the nozzle exit. These conditions are

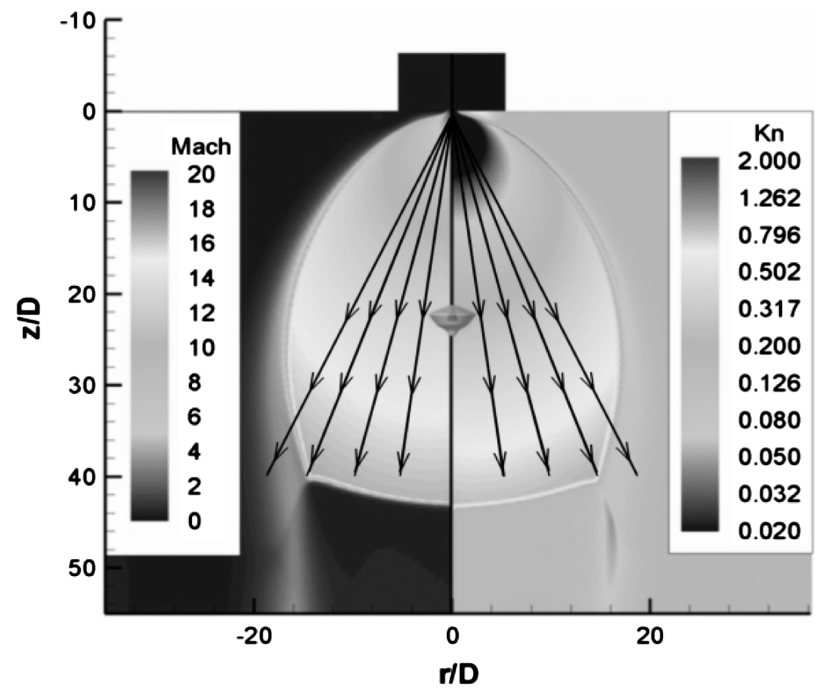

Fig. 4 Ashkenas and Sherman [16] boundary conditions and position of model in test section.

nondimensionalized using the thrust coefficient, as defined by McGhee [18], in order to compare the results with other previous and ongoing work $[\underline{6}, \underline{18}]$. The thrust coefficient of a nozzle is defined as the ratio of the thrust produced by the nozzle to the product of the freestream dynamic pressure and the aeroshell frontal area. The thrust force can be expressed in terms of dynamic and static pressures, as shown in Eq. (3):

$$
C_{T}=\frac{F_{T}}{q_{\mathrm{ref}} \cdot S}=\frac{1}{q_{\mathrm{ref}} \cdot S}\left(2 q_{\mathrm{jet}}+P_{\mathrm{jet}}-P_{\mathrm{amb}}\right) A_{\mathrm{jet}}
$$

The ambient static pressure $P_{\text {amb }}$ in Eq. (3) is the post-bow-shock pressure. Table $\underline{2}$ shows the boundary conditions for the thrust coefficients that are used in this study. The Reynolds number in Table 2 is computed using the conditions at the nozzle exit. The flow from the PD nozzle is assumed laminar, since the jet Reynolds number for all the cases is less than $10^{4}$ [19].

Because of the symmetry of the flowfield, axisymmetric simulations are performed using LeMANS in order to reduce the computational cost and complexity of these simulations. Figure $\underline{5}$ shows some of the computational grids that are used in this study. These meshes are adapted by hand from previous simulations to align the upstream boundary of the computational domain with the bow shock. The grids are structured with quadrilateral elements, because the numerical results are sensitive to the alignment of the grid with the bow shock. Cells are clustered near the wall and in the vicinity of the PD jet in front of the aeroshell. The grid size varies from about

Table 1 Reference freestream conditions

\begin{tabular}{lc}
\hline \hline Parameter & Value \\
\hline$M_{\text {ref }}$ & 12 \\
$\rho_{\text {ref }}, \mathrm{kg} / \mathrm{m}^{3}$ & $4 \times 10^{-4}$ \\
$U_{\text {ref }}, \mathrm{m} / \mathrm{s}$ & 776 \\
$R e_{\text {ref }}$ & 1200 \\
\hline \hline
\end{tabular}

Table 2 PD jet boundary conditions

\begin{tabular}{cccc}
\hline \hline$C_{T}$ & $P_{0, \text { jet }} / P_{0}$ & $\dot{m}, \mathrm{~kg} / \mathrm{s}\left(\times 10^{-6}\right)$ & $R e_{\text {jet }}$ \\
\hline 0.25 & 0.06 & 4 & 700 \\
0.5 & 0.11 & 9 & 1400 \\
1.0 & 0.22 & 18 & 2900 \\
1.5 & 0.33 & 28 & 4300 \\
2.0 & 0.44 & 37 & 5700 \\
2.5 & 0.55 & 46 & 7100 \\
\hline \hline
\end{tabular}




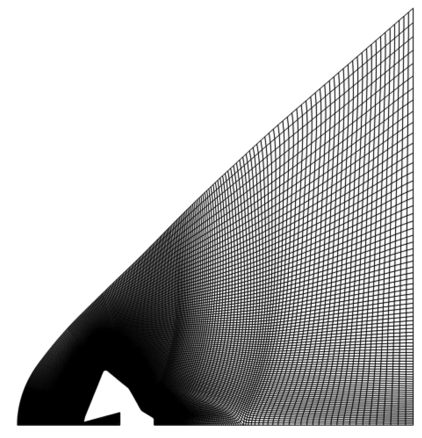

a) $C_{T}=0.5$

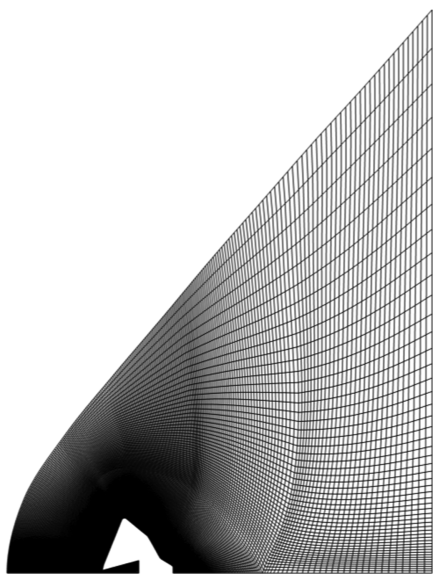

b) $C_{T}=1.5$

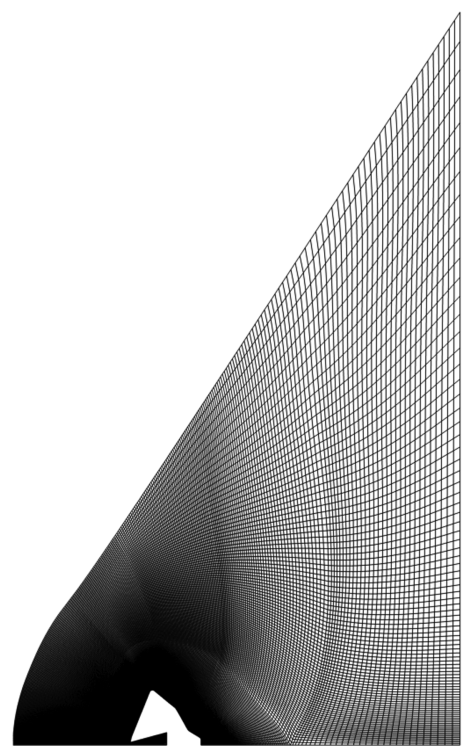

c) $C_{T}=2.5$

Fig. 5 Computational grids.

85,000 cells for the $C_{T}=0.5$ case to approximately 100,500 cells for the $C_{T}=2.5$ case. The average computational runtime for these simulations is approximately 240 CPU hours.

\section{Results}

The goal of this study is to understand the effects of sonic PD jets in a single-nozzle configuration on the flowfield, surface, and aerodynamic properties of a Mars entry aeroshell using the CFD code LeMANS. The numerical results are also used in comparisons with experimental data from previous and ongoing work to assess the computational method. The flowfield properties that are used to study the effects of these parameters are the Mach number and the PD jet species mole fraction. The surface properties are presented as nondimensionalized pressure and skin friction coefficients defined by Eqs. (4) and (5), respectively:

$$
\begin{aligned}
C_{P} & =\frac{P}{(1 / 2) \rho_{\mathrm{ref}} \cdot U_{\mathrm{ref}}^{2}} \\
C_{f} & =\frac{\tau}{(1 / 2) \rho_{\mathrm{ref}} \cdot U_{\mathrm{ref}}^{2}}
\end{aligned}
$$

where $P$ and $\tau$ are the pressure and shear stress along the surface of the aeroshell, respectively. The drag coefficient, given in Eq. (6), is used to investigate the aerodynamic effects,

$$
C_{D}=\frac{F_{D}}{(1 / 2) \rho_{\mathrm{ref}} \cdot U_{\mathrm{ref}}^{2} \cdot S}
$$

The drag force is calculated by integrating the pressure and shear stress over the surface of the aeroshell, excluding the nozzle walls.

\section{A. Flowfield Effects}

Figure 6 presents Mach number contours for the 0.5 thrust coefficient conditions (bottom half is a reflection of the top half). The PD jet expands from sonic conditions at the nozzle exit to higher Mach numbers (i.e., supersonic). The flow then (first) decelerates from supersonic to subsonic velocities through a jet shock, and then from subsonic to zero velocity at a stagnation point detached from the surface of the aeroshell. The freestream also decelerates from hypersonic to subsonic velocities through a bow shock and then to zero velocity at the same stagnation point. In the interface region (region between the bow and jet shocks), the total pressures for the two streams are equal as they both flow outward between the two shocks with subsequent reacceleration to supersonic velocities. The figure also shows a region of separated flow between the PD jet boundary, the surface of the model, and the mixed outflow, with a reattachment point near the shoulder of the aeroshell.

Mach number contours for PD nozzle thrust coefficient values of 0.5, 1.0, 2.0, and 2.5 are shown in Fig. 7. The figure shows that the PD jet expands from sonic conditions at the nozzle exit to supersonic conditions for all four thrust coefficient values. The PD jet then decelerates to zero velocity at a detached stagnation point: first through a shock and then subsonically. The figure also shows that all of the flowfield features shown in Fig. 6 are affected by the nozzle thrust coefficient. The bow shock, interface region, and jet shock move upstream as the thrust coefficient increases in order to equalize the stagnation pressure of the PD jet and the freestream flow. The recirculation region in front of the aeroshell also decreases in size and moves downstream toward the shoulder as the thrust coefficient increases. The reason for this is that the PD jet expands more as the thrust coefficient increases and, therefore, can overcome the relatively sharp turning angle.

Contours of the PD jet mole fraction (i.e., tagged $\mathrm{N}_{2}$ species) for nozzle thrust coefficient values of $0.5,1.0,2.0$, and 2.5 are presented in Fig. $\underline{8}$. As expected, the size of the PD jet increases with the thrust

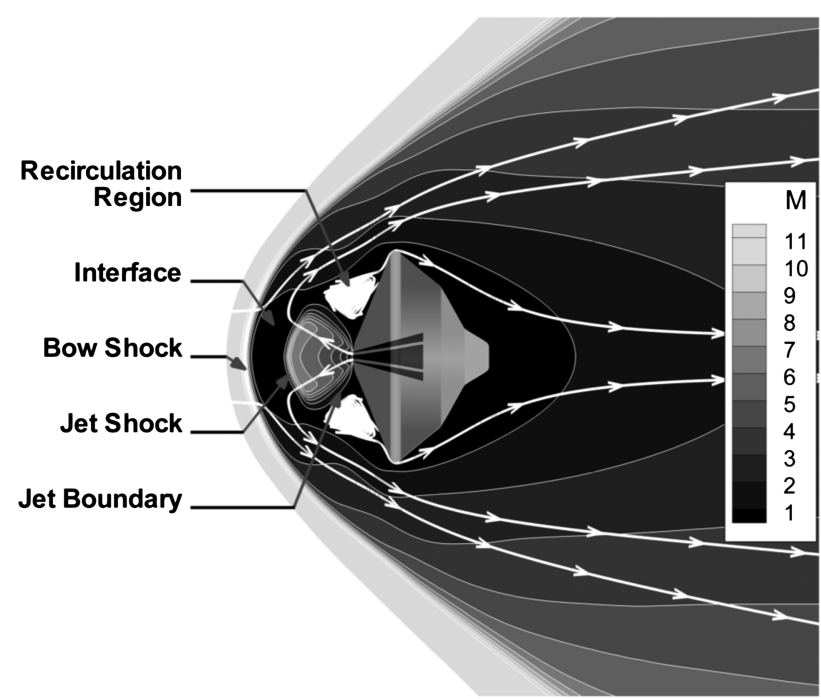

Fig. 6 PD jet flowfield features for $C_{T}=0.5$. 

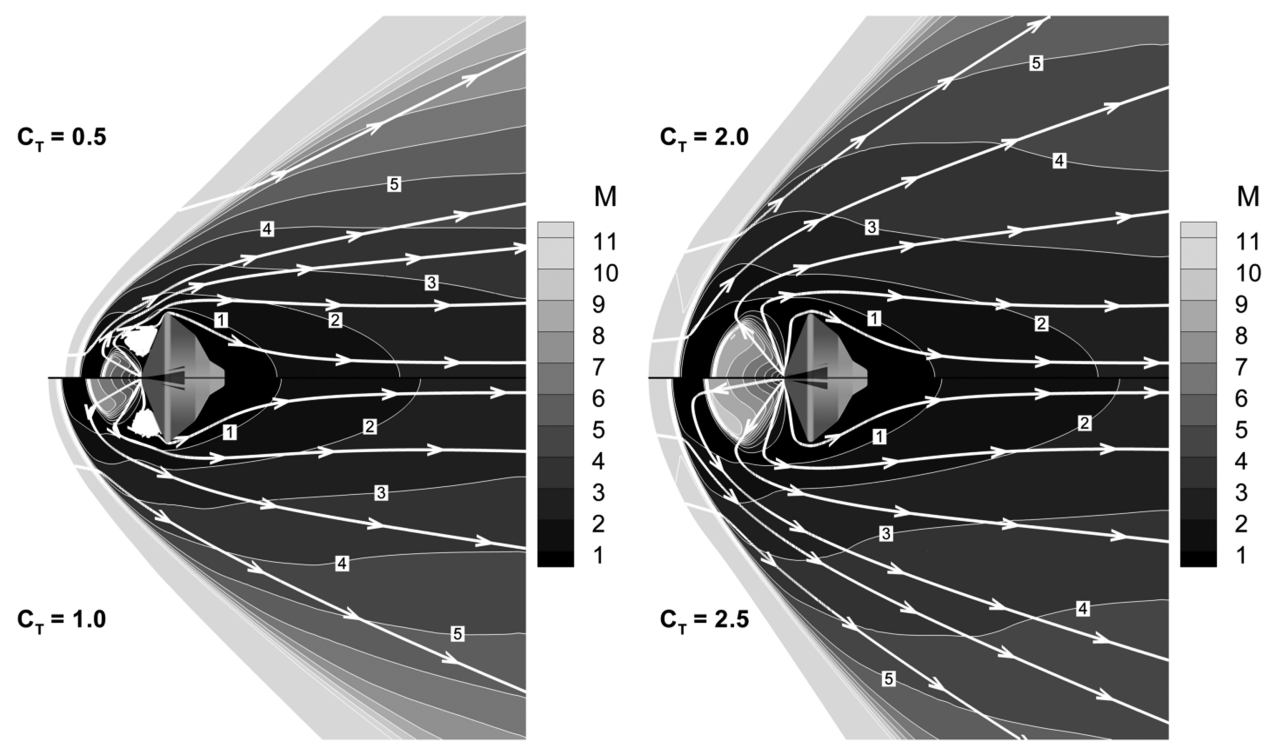

Fig. 7 Mach number contours as function of thrust coefficient.

coefficient. The width of the PD jet grows from approximately half the length of the aeroshell diameter for a thrust coefficient of 0.5 to over a diameter length for a thrust coefficient of 2.5. The amount of PD jet species in the wake also increases as the thrust coefficient increases, since more species are transported downstream by the main flowfield as the mass flow rate of the PD jet increases. This may be significant in the design of the thermal protection system of the aeroshell aftbody, which will need to consider the hot gases of the PD jet.

\section{B. Surface Effects}

Figure $\underline{9}$ presents the pressure and skin friction coefficients along the surface of the aeroshell for thrust coefficients of $0.5,1.0,2.0$, and 2.5 , as well as for the PD jet-off case for comparison. The jet-off case uses a clean geometry without the nozzle cavity. The figure shows that both the surface pressure and shear stress are affected by the thrust coefficient. The pressure along the surface first decreases from a high value near the nozzle exit. The pressure then increases to a peak near the shoulder of the aeroshell and sharply decreases before finally reaching a roughly constant small value along the aftbody. The magnitude of this peak decreases, and the pressure along the forebody approaches an almost constant value equal to the aftbody value as the thrust coefficient increases. The coefficient of skin friction profiles also shows that the shear stress along the surface first decreases from a maximum value at the nozzle exit. The shear stress then decreases to negative values for thrust coefficients less than approximately 2.0 (i.e., flow reattachment begins near $C_{T}=2.0$ ) and then increases to a peak at the shoulder before decreasing and finally reaching an almost constant small value along the aftbody. Similar to the pressure, the overall magnitudes of the coefficient of skin friction approach a roughly constant value along most of the surface as the thrust coefficient increases. The effect of thrust coefficient on the surface properties suggests that the aerodynamic properties of the aeroshell (in particular, the drag force) are also affected.

The overall decrease in pressure along the surface of the aeroshell with increasing thrust coefficient is caused by a shielding effect of the PD jet in the central single-nozzle configuration. This shield prevents mass and momentum from the main freestream flow from reaching the surface of the aeroshell. As the PD jet expands from the nozzle, it pushes the main freestream flow upstream and creates a low pressure region between the jet boundary and the surface. As the thrust coefficient increases, the size of this region also increases, since the size of the PD jet also increases. The location of the peak in the pressure and shear stress profiles near the aeroshell shoulder, shown in Fig. 9, corresponds to the point at which the shielding effect of the
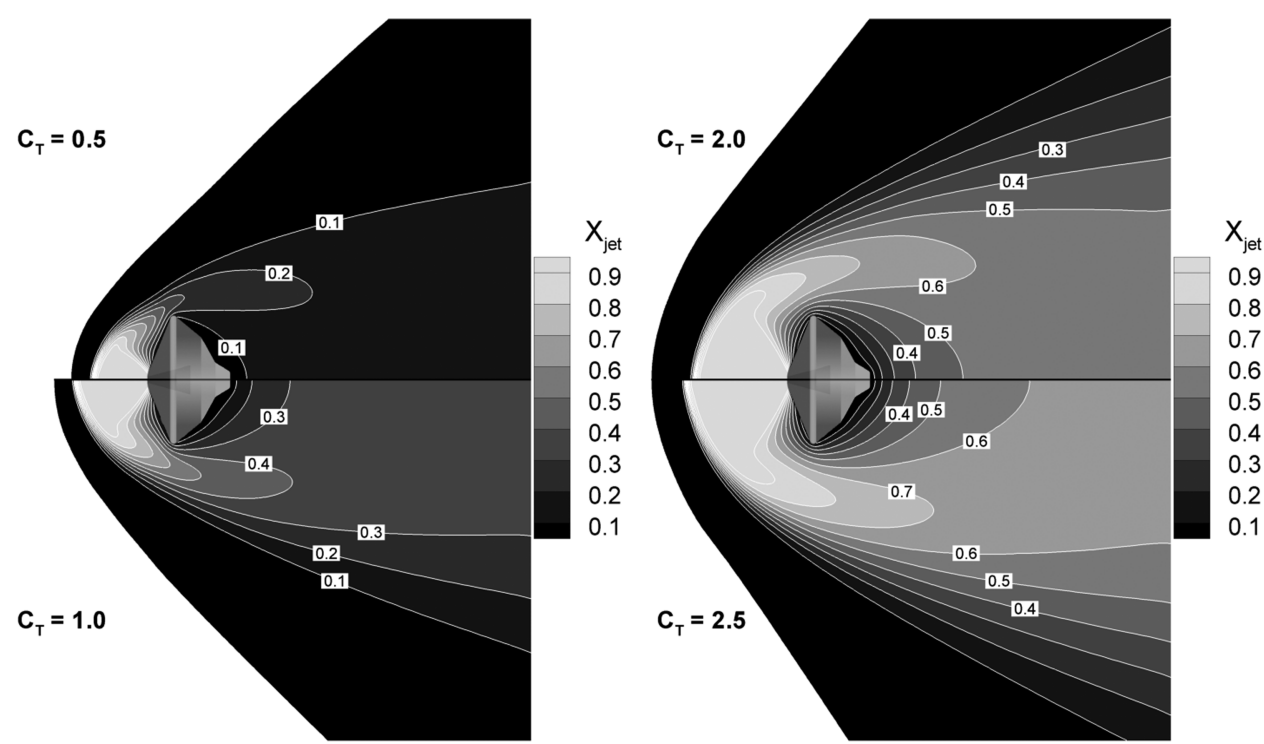

Fig. 8 PD jet species mole fraction contours as function of thrust coefficient. 


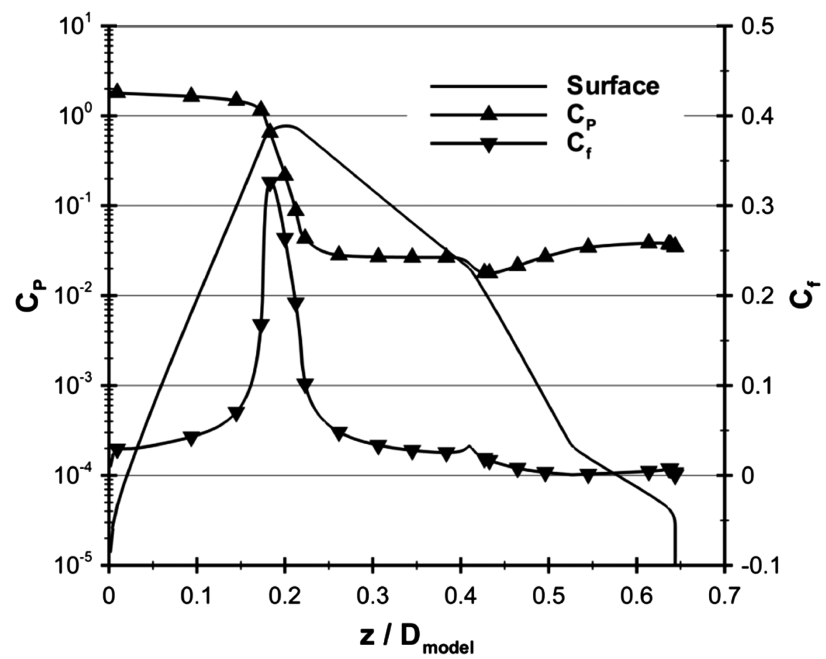

a) $C_{T}=\mathbf{0 . 0}$
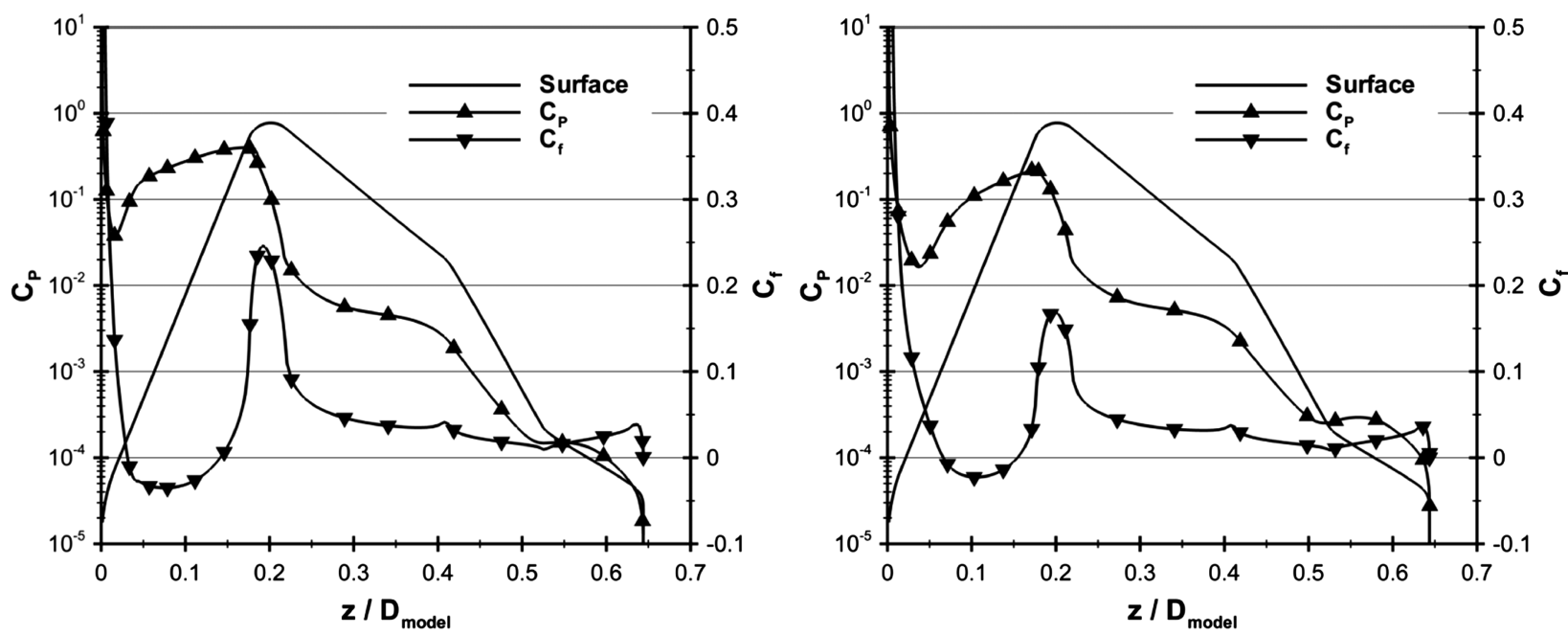

b) $C_{T}=0.5$

c) $C_{T}=1.0$
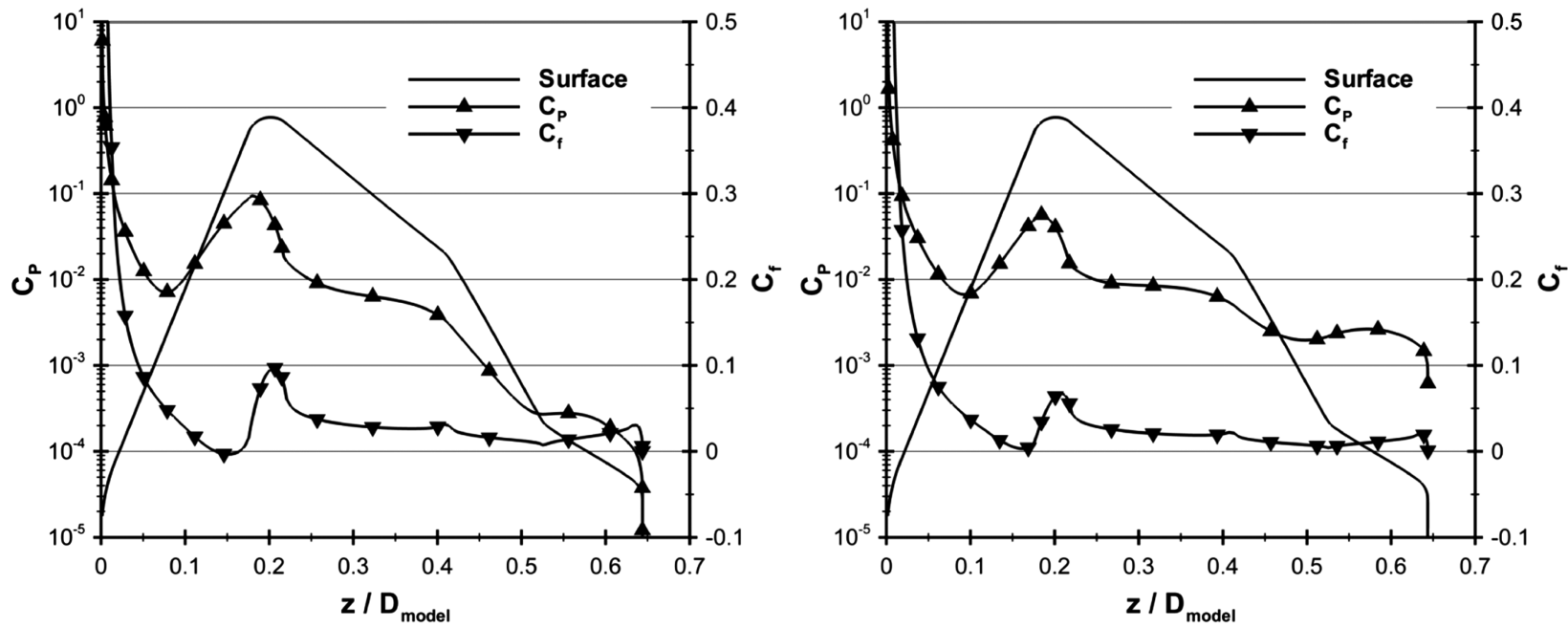

d) $C_{T}=\mathbf{2 . 0}$

e) $C_{T}=2.5$

Fig. 9 Pressure and skin friction coefficient along surface of aeroshell. 


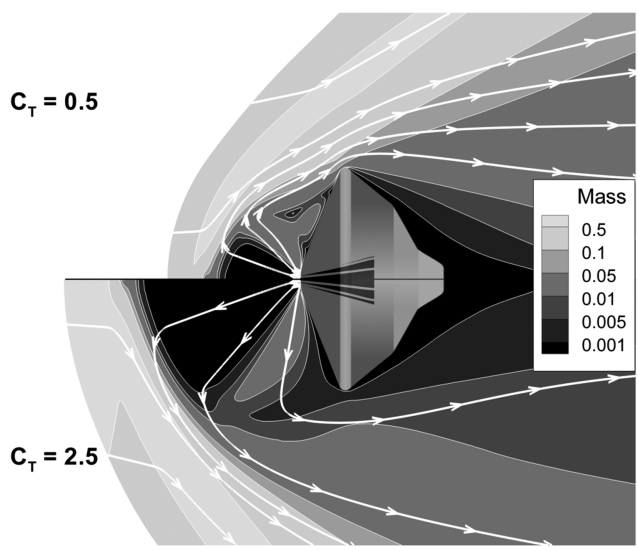

a) Mass $\left[\mathrm{kg} / \mathrm{s} \cdot \mathrm{m}^{2}\right]$

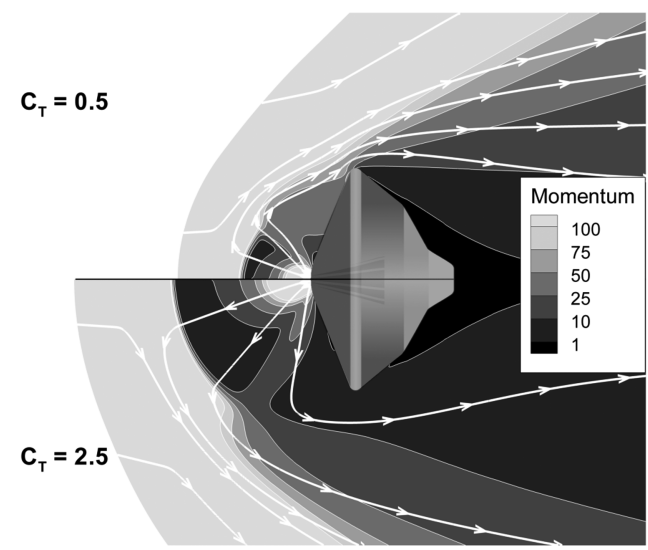

b) Momentum $\left[\mathrm{N} / \mathrm{m}^{2}\right]$

Fig. 10 Mass and momentum transfer from freestream to surface of aeroshell as a function of thrust coefficient.

PD jet becomes negligible. The magnitude of the peak is roughly equal to the value for the jet-off case shown in Fig. 9a. Figure 10 shows mass flux $(\rho U)$ and momentum flux $\left(P+\rho \bar{U}^{2}\right)$ contours computed using the density of the main freestream flow (i.e., excluding the PD jet) for thrust coefficient values of 0.5 and 2.5 in order to quantify the amount of mass and momentum transferred to the surface. The figure shows that less mass and momentum from the freestream reach the surface of the aeroshell as the thrust coefficient increases, which decreases the overall surface pressure values.

\section{Aerodynamic Effects}

Figure 11 presents the aerodynamic drag coefficient, computed using Eq. ( 6 ), as a function of thrust coefficient. The figure also shows the total axial force coefficient of the aeroshell, which is equal to the sum of the aerodynamic drag and thrust coefficients. As the thrust coefficient increases, the drag coefficient decreases and asymptotically approaches a constant value that is approximately

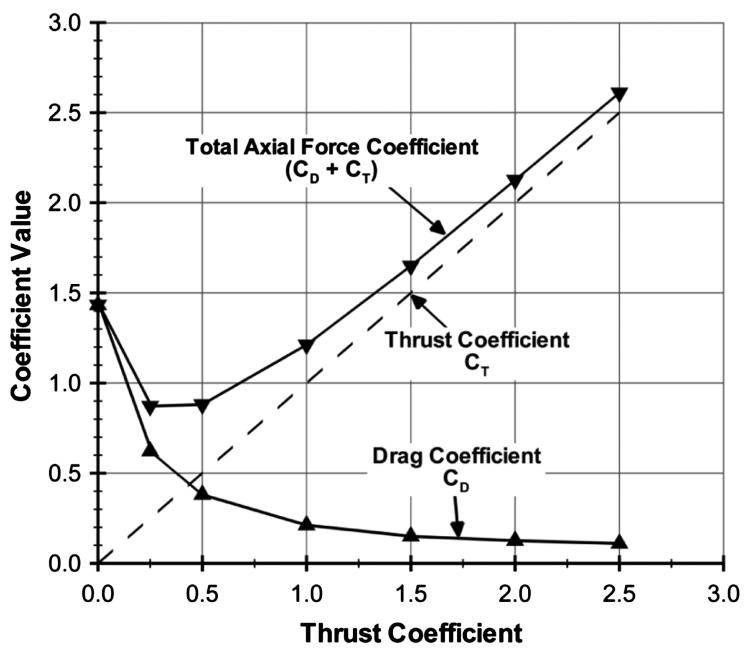

Fig. 11 Drag and total axial force coefficients.
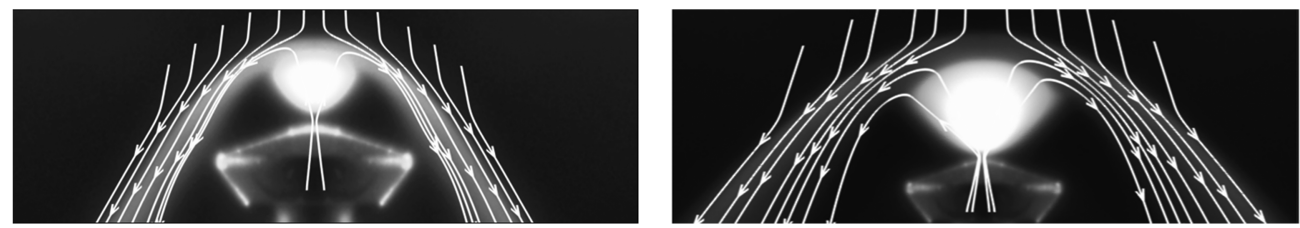

a) $C_{T}=0.5$

b) $C_{T}=2.0$

Fig. 12 Bow shock profile comparison (images: PLIIF; lines: LeMANS velocity streamlines). equal to $8 \%$ of the value for the PD jet-off case $\left(C_{D}=1.4\right.$ for $C_{T}=0$, and $C_{D}=0.11$ for $C_{T}=2.5$ ). The decrease in the aerodynamic drag coefficient with increasing thrust coefficient is due to lower surface pressure along the aeroshell forebody. The figure also shows that the total axial force coefficient first decreases as the thrust coefficient increases, and then it begins to increase for thrust coefficient values greater than approximately 0.5 . The total axial force coefficient does not exceed the drag coefficient for the PD jet-off case until the thrust coefficient is equal to about 1.25 , where most of the contribution to the axial force is from the PD thrust. This suggests that propulsive deceleration using sonic PD jets in a single-nozzle configuration is only beneficial for relatively large thrust coefficient values that are greater than approximately 1.25 . The total axial force coefficient increases by roughly constant increments for thrust coefficients greater than 1.25 , since the drag coefficient is almost constant.

\section{Comparison with Experimental Data}

Comparisons between the numerical results and experimental data obtained from previous and current work are carried out to assess the computational method. The images in Fig. 12 are PLIIF visualizations, where the bright areas represent regions with relatively high density values. The lines in the figure are velocity streamlines computed from the numerical results. The figure shows good qualitative agreement between LeMANS and PLIIF with respect to the bow shock profile around the aeroshell.

Figure 13a shows the bow shock standoff distance (i.e., the distance from the center of the aeroshell forebody to the bow shock along the stagnation streamline) for LeMANS and PLIIF. This distance corresponds to the location where the density begins to increase at the bow shock. As can be seen from the figure, the numerical results are in good agreement with the experimental data. Although small (less than 3\%), the difference in the standoff distance between the numerical and experimental results can be attributed to the fact that this distance was measured in the experiments using the fluorescence signal, which is known to be a function of the iodine number density and rotational temperature. For the numerical results, however, the standoff distance was calculated using only the iodine number density. The aerodynamic properties calculated using LeMANS are also compared with experimental data obtained by 


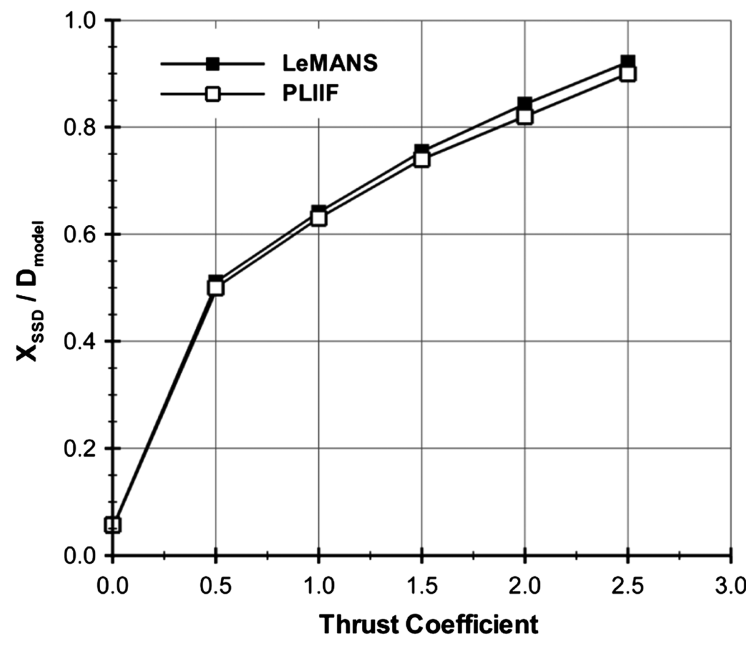

a) Bow shock standoff distance

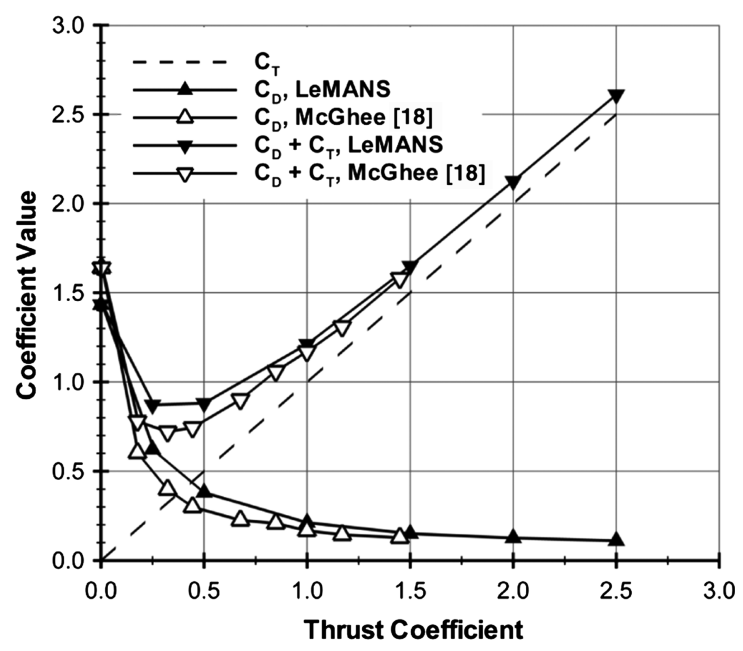

b) Aerodynamic coefficient

Fig. 13 Comparison of bow shock standoff distance and aerodynamic coefficients between computational and experimental data.

McGhee [18] in the early 1970s. This experimental work investigated the aerodynamic interactions of supersonic PD jets $\left(M_{\text {jet }}=3.0\right)$ in supersonic flow $\left(M_{\infty}=6.0\right)$ using a $70^{\circ}$ blunt-cone geometry with a central single-nozzle PD jet. Although the freestream and PD jet Mach numbers are different between the present computations and the experimental study, Fig. 13b shows overall good agreement between the two sets of results in terms of the aerodynamic effects of single-nozzle PD jets. Both sets of results show similar trends and very close values for the aerodynamic drag and total axial force coefficients.

\section{Conclusions}

The purpose of this study was to investigate the interactions of a single-nozzle sonic PD jet on Mars entry aeroshells. The paper described numerical and experimental methods that were used to understand these complex flow interactions. Using the CFD code LeMANS, the effects on the flowfield, surface, and aerodynamic properties around an MSL-based aeroshell were evaluated for Mach 12 flow of $\mathrm{I}_{2}$-seeded $\mathrm{N}_{2}$ gas using axisymmetric laminar simulations. A sonic nozzle was placed at the center of the aeroshell forebody to supply the PD jet. The boundary conditions for this jet were specified using a nondimensional nozzle thrust coefficient in order to be able to compare the numerical results with experimental data from previous and ongoing work. The first part of this study focused on the flowfield effects of the PD jet. The results showed that, as the thrust coefficient increases, the bow shock, jet shock, and interface region move upstream, while the recirculation region in front of the aeroshell moves downstream toward the shoulder and becomes smaller in size. The results also showed that the size of the PD jet increases, and more jet species are transported to the wake as the thrust coefficient increases. The second part of this study examined the effects of the PD jet on the surface properties of the aeroshell. The numerical results showed that the overall magnitudes of the pressure and shear stress approach a roughly constant value along most of the surface as the thrust coefficient increases. In the third section of the study, the aerodynamic effects of the PD jet were investigated. It was found that the aerodynamic drag decreases and asymptotically nears a constant value of $8 \%$ of the jet-off case as the thrust coefficient increases. This is caused by a shielding effect of the PD jet, which prevents mass and momentum from the main freestream flow from reaching the surface of the aeroshell. The results also showed that the total axial force coefficient (i.e., the sum of the aerodynamic drag and thrust coefficients) does not exceed the drag coefficient for the jet-off case until a thrust coefficient of 1.25 , where most of the contribution is from the PD thrust. In the last section of this study, comparisons between LeMANS and experimental data showed good agreement in the bow shock profile and standoff distance, as well as the aerodynamic coefficients.

\section{Acknowledgments}

The authors gratefully acknowledge funding for this work through NASA Research Announcement grant NNX08AH37A. The use of supercomputers at the University of Michigan (Center for Advanced Computing) and NASA (NASA Advanced Supercomputing Division) is essential to this work and is also greatly appreciated.

\section{References}

[1] Edquist, K. T., Dyakonov, A. A., Wright, M. J., and Tang, C. Y., "Aerothermodynamic Environments Definition for the Mars Science Laboratory Entry Capsule," AIAA Paper 2007-1206, Jan. 2007.

[2] Braun, R. D., and Manning, R. M., "Mars Exploration Entry, Descent, and Landing Challenges," Journal of Spacecraft and Rockets, Vol. 44, No. 2, March-April 2007, pp. 310-323. doi: $10.2514 / 1.25116$

[3] Korzun, A. M., Braun, R. D., and Cruz, J. R., "Survey of Supersonic Retropropulsion Technology for Mars Entry, Descent, and Landing," Journal of Spacecraft and Rockets, Vol. 46, No. 5, Sept.-Oct. 2009, pp. 929-937. doi: $10.2514 / 1.41161$

[4] McDaniel, J. C., Glass, C. E., Staack, D., and Miller, C. G., "Experimental and Computational Comparisons of an Underexpanded Jet Flowfield," AIAA Paper 2002-0305, Jan. 2002.

[5] Cecil, D. E., and McDaniel, J. C., "Planar Laser-Induced Iodine Fluorescence Measurements in Rarefied Hypersonic Flow," Proceedings of the 24th International Symposium on Rarefied Gas Dynamics, American Inst. of Physics, New York, 2005, pp. 1325-1350.

[6] Reed, E. M., Codoni, J., McDaniel, J. C., Alkandry, H., and Boyd, I. D., "Investigation of the Interactions of Reaction Control Systems with Mars Science Laboratory Aeroshell," AIAA Paper 2010-1558, Jan. 2010

[7] Scalabrin, L. C., and Boyd, I. D., "Development of an Unstructured Navier-Stokes Solver for Hypersonic Nonequilibrium Aerothermodynamics," AIAA Paper 2005-5203, June 2005.

[8] Scalabrin, L. C., and Boyd, I. D., "Numerical Simulation of Weakly Ionized Hypersonic Flow for Reentry Configurations," AIAA Paper 2006-3773, June 2006.

[9] Scalabrin, L. C., and Boyd, I. D., "Numerical Simulations of the FIREII Convective and Radiative Heating Rates," AIAA Paper 2007-4044, June 2007.

[10] Holman, T. D., and Boyd, I. D., "Effects of Continuum Breakdown on the Surface Properties of a Hypersonic Sphere," Journal of Thermophysics and Heat Transfer, Vol. 23, No. 4, Oct.-Dec. 2009, pp. 660-673.

doi: $10.2514 / 1.43509$

[11] Wilke, C. R., "A Viscosity Equation for Gas Mixtures," Journal of 
Chemical Physics, Vol. 18, No. 4, 1950, pp. 517-519. doi:10.1063/1.1747673

[12] Blottner, F. G., Johnson, M., and Ellis, M., "Chemically Reacting Viscous Flow Program for Multi-Component Gas Mixtures," Sandia Labs., TR SC-RR-70-754, Albuquerque, NM, 1971.

[13] Vincenti, W. G., and Kruger, C. H., Introduction to Physical Gas Dynamics, Krieger, Malabar, FL, 2002, p. 21.

[14] MacCormack, R. W., and Candler, G. V., "The Solution of the NavierStokes Equations Using Gauss-Seidel Line Relaxation," Computers and Fluids, Vol. 17, No. 1, 1989, pp. 135-150. doi:10.1016/0045-7930(89)90012-1

[15] Karypis, G., and Kumar, V., METIS: A Software Package for Partitioning Unstructured Graphs, Partitioning Meshes, and Computing Fill-Reducing Orderings of Sparse Matrices, Univ. of Minnesota, Minneapolis, MN, 1998.
[16] Ashkenas, H., and Sherman, F. S., "The Structure and Utilization of Supersonic Free Jets in Low Density Wind Tunnels," Proceedings of the 4th International Symposium on Rarefied Gas Dynamics, Academic Press, New York, 1966, pp. 84-105.

[17] Alkandry, H., Boyd, I. D., Reed, E. M., and McDaniel, J. C., "Numerical Study of Hypersonic Wind Tunnel Experiments for Mars Entry Aeroshells," AIAA Paper 2009-3918, June 2009.

[18] McGhee, R. J., "Effects of a Retronozzle Located at the Apex of a 140 Degree Blunt Cone at Mach Numbers of 3.00, 4.50, and 6.00," NASA TN D-6002, Jan. 1971.

[19] Pope, S. B., Turbulent Flows, Cambridge Univ. Press, New York, 2000, pp. $96-102$.

R. Cummings Associate Editor 